\title{
BALANÇO DE CARBONO EM MONOCULTIVO DE EUCALIPTO COM DIFERENTES ARRANJOS ESPACIAIS
}

\author{
Daniel Brianezi ${ }^{1}$, Laércio Antônio Gonçalves Jacovine², Bruno Leão Said Schettini² ${ }^{2}$ Carlos Moreira \\ Miquelino Eleto Torres², Samuel José Silva Soares da Rocha², Paulo Henrique Villanova², Silvio \\ Nolasco de Oliveira Neto ${ }^{2}$
}

\begin{abstract}
RESUMO - As plantações de eucalipto podem compensar as emissões de gases com efeito estufa (GEE) das atividades de fertilização e plantio, devido à capacidade das árvores em armazenar carbono na forma de biomassa. O objetivo deste estudo foi estimar o sequestro de carbono em plantações de eucalipto com diferentes densidades de instalação. O estudo foi conduzido no município de Lamim, no estado de Minas Gerais - Brasil, numa plantação de eucalipto dividida em quatro áreas de 0,25 ha com diferentes densidades de instalação. O diâmetro à altura do peito e altura total dos indivíduos foram obtidos por inventário florestal. A biomassa foi calculada pela multiplicação do volume e densidade básica da madeira. As emissões de GEE foram calculadas a partir dos dados de fertilização e calagem. O reservatório de carbono (90,94 $\mathrm{MgCO}_{2 \mathrm{eq}}$ ha-1) e as emissões de GEE foram superiores no espaçamento de $2 \times 1 \mathrm{~m}$. A menor emissão de GEE por $\mathrm{m}^{3}$ de madeira produzido foi no espaçamento $2 \times 2 \mathrm{~m}$. O balanço de carbono foi positivo em todos os espaçamentos avaliados. Foi possível concluir que as florestas de Eucalipto são capazes de compensar as emissões de GEE da fertilização nitrogenada e calagem em plantações pouco tecnificadas.
\end{abstract}

Palavras chave: acordo de Paris, agricultura de baixo carbono, gases de efeito estufa.

\section{CARBON BALANCE IN EUCALYPTUS MONOCULTURE WITH DIFFERENT SPACE ARRANGEMENTS}

\begin{abstract}
Eucalyptus monocultures may be able to neutralize greenhouse gas (GHG) emissions resulted from fertilization and planting activities due to trees' ability to store carbon in the form of biomass. Thus, the objective of the study was to quantify the carbon balance in Eucalyptus plantation under different spacings arrangements. The study was conducted in the municipality of Lamim, in the state of Minas Gerais - Brazil, in a plantation divided into four areas of 0.25 ha with different tree spacings $(2 \times 1 \mathrm{~m}, 2 \times 2 \mathrm{~m}, 3 \times 2 \mathrm{~m}$ $e 3 \times 3 \mathrm{~m}$ ). The diameter at breast height and total height of the individuals were obtained by forest inventory. Biomass was calculated by multiplying the volume and basic density of the wood. GHG emissions resulted from management operations were calculated from nitrogen fertilization and liming data. The carbon stock (90.94 $\mathrm{MgCO}_{2 e q} \mathrm{ha}^{-1}$ ) and GHG emissions were higher at 2 × $1 \mathrm{~m}$ spacing. The lowest GHG emission per $\mathrm{m}^{3}$ of wood produced was in the $2 \times 2 \mathrm{~m}$ spacing. The carbon balance was positive in the spacings arrangements evaluated. We conclude that Eucalyptus forests are able to neutralize GHG emissions from nitrogen fertilization and liming in low-tech plantations.
\end{abstract}

Keywords: greenhouse gases, low carbon agriculture, Paris agreement.

\footnotetext{
${ }^{1}$ Centro Federal de Educação Tecnológica de Minas Gerais (CEFET - MG) - danielbrianezi@gmail.com ;

${ }^{2}$ Departamento de Engenharia Florestal - Universidade Federal de Viçosa (UFV).
} 


\section{INTRODUÇÃO}

O aumento das emissões de Gases de Efeito Estufa (GEE) causam as mudanças climáticas em escala global. Limitar essas alterações do clima tem motivado negociações internacionais ao longo dos últimos anos (Riahy et al., 2015). Em 1995, iniciaram as Conferências das Partes (Conference of the Parties - COP), que são reuniões internacionais periódicas que têm como objetivo gerar políticas públicas que permitam minimizar as consequências gerados com o aumento das emissões de GEE (NEEFF, 2013). Na COP 21, realizada em Paris no ano de 2015, foi elaborado o acordo de Paris, que prevê limitar o aumento da temperatura terrestre em $2^{\circ} \mathrm{C}$ em relação aos níveis pré-industriais (Ghezloun et al., 2017).

O Brasil é um dos países signatários do acordo de Paris com a meta de redução das emissões de GEE em $37 \%$ até ao ano de 2025, em relação aos níveis de 2005, com ações nos principais setores da economia do país. No setor florestal, os principais objetivos são a implementação do código florestal brasileiro em toda a extensão do país, já que o código foi alterado pela Lei ${ }^{\circ} 12.651 / 2012$. Além disso existe o incentivo a implantação de novas áreas com florestas plantadas e a restauração de 12 milhões de hectares de florestas até 2030 no país (BRASIL, 2015).

A área de florestas plantadas no Brasil totalizou 7,84 milhões de hectares no ano de 2016. A principal espécie cultivada no país é do gênero Eucalyptus sp., que representou $72 \%$ desse total (Ibá, 2017). Por se tratar de uma espécie de crescimento rápido, estas florestas têm um importante papel na mitigação das mudanças climáticas devido à capacidade das árvores absorver dióxido de carbono da atmosfera e armazenálo na forma de biomassa florestal (Susaeta et al., 2017).

A escolha do espaçamento de plantio influencia o desenvolvimento das plantas (Bouillet et al., 2013) e, como o crescimento está diretamente relacionada com a acumulação de biomassa (Trotsiuk et al., 2016), o que permite favorecer o reservatório de carbono de uma floresta. A aplicação de fertilizantes nitrogenados e calcário são práticas frequentemente utilizadas na condução e desenvolvimento deste tipo de espécies florestais (Vieira et al., 2015). Porém contribuem para o aumento das emissões de GEE (Kunhikrishnan et al., 2016).
As florestas podem ser capazes de neutralizar as emissões de GEE das atividades do plantio e da fertilização (Proietti et al., 2016), mas o seu comportamento pode variar de acordo com os diferentes arranjos espaciais adotados. Dessa forma, o objetivo do estudo foi realizar o balanço de carbono em um monocultivo de eucalipto sob diferentes espaçamentos e avaliar as emissões de GEE por $\mathrm{m}^{3}$ de madeira produzido em cada um deles.

\section{MATERIALE MÉTODOS}

\section{Área de estudo}

O estudo foi desenvolvido em uma propriedade rural localizada na cidade de Lamim, Zona da Mata de Minas Gerais, sob as coordenadas geográficas $20^{\circ}$ 47' 24' S e 43 $28^{\circ} 26^{\prime \prime} \mathrm{O}$, em um plantio de Eucalipto (com 32 meses de idade) pouco tecnificado, que são aqueles realizados em propriedades rurais de pequeno porte, com pouco recurso financeiro e na sua maioria sem o uso de máquinas agrícolas. O componente arbóreo é um hibrido de Eucalyptus urophylla x Eucalyptus grandis, plantados em diferentes arranjos espaciais (Quadro 1). O plantio foi dividido em quatro áreas de 0,25 ha com espaçamentos de $2 \times 1 \mathrm{~m}$ (1250 plantas), 2 × 2 m (625 plantas), 3 × 2 m (417 plantas) e 3 × 3 m (278 plantas).

A região é composta por relevo montanhoso (72\%), ondulado (21\%) e plano (7\%), com encostas convexocôncavas embutidas em vales de fundo chato, formados por terraços e leitos maiores, onde se localizam cursos d'água (COELHO et al., 2008). O solo local é classificado como Latossolo Vermelho-Amarelo Distrófico, pobre em bases trocáveis e de baixa fertilidade natural conforme análise de solo feita na área de estudo. O clima da região é do tipo Cwb, temperado úmido com inverno seco e verão temperado, segundo o sistema de Köppen. A temperatura média anual é de $19,3^{\circ} \mathrm{C}$, altitude média

Quadro 1 - Código da área, espaçamento (em m), número de parcelas e área das parcelas do plantio avaliados $\left(\mathrm{em} \mathrm{m}^{2}\right)$

\begin{tabular}{cccc}
\hline Área & $\begin{array}{c}\text { Espaçamento } \\
(\mathrm{m})\end{array}$ & $\begin{array}{c}\text { Número } \\
\text { de parcelas }\end{array}$ & $\begin{array}{c}\text { Área das } \\
\text { parcelas }\left(\mathrm{m}^{2}\right)\end{array}$ \\
\hline 1 & $2 \times 1$ & 4 & 48 \\
2 & $2 \times 2$ & 4 & 96 \\
3 & $3 \times 2$ & 3 & 144 \\
4 & $3 \times 3$ & 3 & 216 \\
\hline
\end{tabular}


de 779m e a precipitação média anual de 1.300 mm (Alvares et al., 2013).

\section{Inventário florestal}

O diâmetro à altura do peito (dap) a 1,30 m do solo e altura total dos indivíduos ( $H t)$ foram obtidos por inventário florestal, 48 árvores (12 por espaçamento) foram selecionadas e submetidas a cubagem rigorosa pelo método não destrutivo. Os diâmetros nas alturas de $0 ; 0,3 ; 0,7 ; 1,3 \mathrm{~m}$ foram medidos e a partir deste, a cada um metro, até um limite de $6,5 \mathrm{~cm}$ mensurado com pentaprisma de Wheeler. O volume total com casca das árvores foi determinado pelo método de Smalian.

\section{Biomassa e carbono}

A biomassa foi calculada pela multiplicação do volume e da densidade básica da mesma, que foi obtida a partir de amostras de madeira retiradas com um trado mecânico, de acordo com a norma ABNT-NBR 11941, em que a densidade da madeira é calculada pelo método da imersão em água. O carbono armazenado nas árvores amostras foi estimado por multiplicação dos valores de biomassa pelo fator 0,47, recomendado para espécies arbóreas pelo Painel Intergovernamental de Mudanças Climáticas - IPCC (2006).

A partir dos dados de estoque de carbono no fuste das árvores amostra foi gerada uma equação, baseadas no modelo de Schumacher \& Hall linearizado, para calcular o estoque de carbono do fuste nas demais árvores do povoamento:

$$
\operatorname{LnY}=\left(\beta_{0}+\beta_{\mathrm{i}}\right) *\left(\operatorname{Lndap}+\beta_{2}\right) *(\mathrm{LnH}+\beta \mathrm{t}+\varepsilon)
$$

Em que: Ln= logaritmo neperiano; $\mathrm{Y}=$ carbono do fuste, em $\mathrm{kgC} ; \beta_{\mathrm{i}}=$ parâmetros do modelo, para $\mathrm{i}=1$, 2 e $3 ; \beta_{0}=-3,9980, \beta_{1}=1,8016, \beta_{2}=0,7559$; e $\varepsilon=$ erro aleatório. $\mathrm{O} \mathrm{R}_{\text {adj }}^{2}=88,38 \%$.

O incremento médio anual em carbono (IMAC) para cada umas das áreas foi obtido pela divisão do estoque de carbono pela idade de plantio (32 meses).

\section{Emissões de Gases de Efeito Estufa (GEE) e por Unidade Produzida}

As emissões de GEE consideradas foram calculadas a partir dos dados de fertilização nitrogenada (150 g cova-1 $^{-1}$ de NPK na formulação 20-00-20) e calagem (0,5 $\mathrm{Mg} \mathrm{ha}^{-1}$ de calcário calcítico). Também foram usados fosfato natural reativo e superfosfato simples, que não emitem GEE e não foram levados em consideração no cálculo. As fertilizações foram realizadas manualmente, não havendo, por isso, emissões de GEE correspondentes.

As emissões totais de óxido nitroso $\left(\mathrm{N}_{2} \mathrm{O}\right)$, pelo uso de fertilizantes, foram calculadas pelo somatório das emissões diretas e emissões indiretas de $\mathrm{N}_{2} \mathrm{O}$.

$$
\mathrm{E}_{\mathrm{N} 2 \mathrm{O}}=\mathrm{E}_{\mathrm{N} 20 \mathrm{~d}}+\mathrm{E}_{\mathrm{N} 20 \mathrm{i}}
$$

Em que: $\mathrm{E}_{\mathrm{N} 2 \mathrm{O}}=$ emissões de $\mathrm{N}_{2} \mathrm{O}\left(\mathrm{em} \mathrm{Mg} \mathrm{ha}^{-1}\right)$; $\mathrm{E}_{\mathrm{N} 2 \mathrm{Od}}=$ emissão de $\mathrm{N}_{2} \mathrm{O}$ direta $\left(\mathrm{em} \mathrm{Mg} \mathrm{ha}^{-1}\right) ; \mathrm{E}_{\mathrm{N} 2 \mathrm{Oi}}=$ emissão de $\mathrm{N}_{2} \mathrm{O}$ indireta (em Mg ha ${ }^{-1}$ ) (IPCC, 2006).

As emissões diretas são provenientes do uso de fertilizantes sintéticos. Já as emissões indiretas de $\mathrm{N}_{2} \mathrm{O}$ referem-se à porção de nitrogênio incorporado no solo que é volatilizada na forma de $\mathrm{NH}_{3}$ e $\mathrm{NO}_{\mathrm{x}}$ ou perdida por lixiviação (IPCC, 2006).

$$
\begin{gathered}
\mathrm{E}_{\mathrm{N} 2 \mathrm{Od}}=\mathrm{Qf} * \mathrm{FE}_{\mathrm{N} 2 \mathrm{Od}} * \mathrm{~F}_{\mathrm{N}-\mathrm{N} 2 \mathrm{Ov}}=\left(\mathrm{Qf} * \mathrm{Frac}_{\mathrm{gas}}\right) \\
* \mathrm{FE}_{\mathrm{Nv}} * \mathrm{FN}_{-\mathrm{N} 2 \mathrm{~V}} \\
\mathrm{E}_{\mathrm{N} 2 \mathrm{Ol}}=\left(\mathrm{Qf} * \mathrm{Fra}_{\mathrm{Clix}}\right) * \mathrm{FE}_{\mathrm{Nl}} * \mathrm{~F}_{\mathrm{N}-\mathrm{N} 2 \mathrm{O}}
\end{gathered}
$$

Em que: $\mathrm{Q}_{\mathrm{f}}=$ quantidade de fertilizante utilizada $\left(\right.$ em $\left.\mathrm{Mg} \mathrm{ha}^{-1}\right)$; Frac $_{\text {gas }}=$ fração de $\mathrm{N}$ volatilizada na forma de $\mathrm{NH}_{3}$ e $\mathrm{NO}_{\mathrm{x}}\left(\mathrm{em} \mathrm{Mg} \mathrm{ha}{ }^{-1}\right.$ ). Foram adotados os seguintes

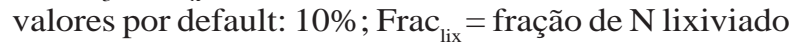
(em $\mathrm{Mg} \mathrm{ha}^{-1}$ ). 30\% para $\mathrm{FE}_{\mathrm{N} 2 \mathrm{Od}}=$ fator de emissão de $\mathrm{N}_{2} \mathrm{O}$ para emissão direta. $1 \%$ para $\mathrm{FE}_{\mathrm{Nv}}=$ fator de emissão de $\mathrm{N}$ volatilizado. $1 \%$ para $\mathrm{FE}_{\mathrm{Nl}}=$ fator de emissão de $\mathrm{N}$ lixiviado. 0,75\% para $\mathrm{F}_{\mathrm{N}-\mathrm{N} 2 \mathrm{O}}=$ Fator de conversão de $\mathrm{N}-\mathrm{N}_{2} \mathrm{O}$ massa específica do $\mathrm{N}_{2} \mathrm{O}$ dividido pela massa específica do $\mathrm{N}(44 / 28)$. Valores esses recomendados pelo IPCC (2006).

As emissões da calagem foram calculadas de acordo com a seguinte equação:

$$
\mathrm{E}_{\mathrm{CO} 2 \mathrm{e}}=\mathrm{Q}_{\mathrm{A}} * \mathrm{FE}_{\text {Calagem }} * 44 / 12
$$

Em que: $\mathrm{E}_{\mathrm{CO} 2 \mathrm{e}}=$ emissão de dióxido de carbono equivalente (em $\mathrm{Mg} \mathrm{ha}^{-1}$ ); $\mathrm{Q}_{\mathrm{A}}=$ quantidade de calcário aplicado (em $\mathrm{Mg} \mathrm{ha}^{-1}$ ); $\mathrm{FE}_{\text {Calagem }}=$ fator de emissão para a calagem. $12 \%$ para $\mathrm{CaCO}_{3}$ (IPCC, 2006).

As emissões por unidade produzidas foram calculadas pela divisão do volume de madeira em cada área por suas respectivas emissões de GEE.

\section{Balanço de Carbono em cada Área}

O balanço de carbono foi calculado por meio dos valores de estocagem e emissões de GEE com a equação: 
$\mathrm{B}_{\mathrm{GEE}}=\mathrm{C}_{\mathrm{CO} 2 \mathrm{e}}-\mathrm{E}_{\mathrm{CO} 2 \mathrm{e}}$; em que: $\mathrm{B}_{\mathrm{GEE}}=$ balanço de remoções e emissões de gases de efeito estufa, em $\mathrm{MgCO}_{2 \mathrm{e}} ; \mathrm{C}_{\mathrm{CO}_{\mathrm{e}}}=$ estoque total de carbono, em $\mathrm{MgCO}_{2 \mathrm{e}}$; $\mathrm{E}_{\mathrm{CO} 2 \mathrm{e}}=$ somatório das emissões de gases de efeito estufa, em $\mathrm{MgCO}_{2 \mathrm{e}}$.

\section{RESULTADOS}

\section{Estoque e incremento médio anual em carbono}

O estoque de carbono médio encontrado para os 4 espaçamentos aos 32 meses foi de 25,39 $\pm 6,68 \mathrm{MgCO}_{2 \mathrm{eq}}$ ha $^{-1}$. Aárea 1 apresentou a maior acumulação de carbono e IMAC (Quadro 2).

\section{Emissões de GEE e balanço de carbono}

As emissões de GEE foram superiores no espaçamento de $2 \times 1 \mathrm{~m}$, seguidos por 2x2 $\mathrm{m}, 3 \times 2 \mathrm{~m}$, $3 \times 3 \mathrm{~m}$, respectivamente. A principal fonte de GEE foi a fertilização nitrogenada, exceto no espaçamento de $3 \times 3 \mathrm{~m}$ em que a calagem foi superior. O balanço de carbono foi positivo em todos os espaçamentos avaliados (Quadro 3).

As emissões por $\mathrm{m}^{3}$ de madeira foram inferiores no espaçamento de $2 \times 2 \mathrm{~m}$, seguidas pelo espaçamento 3x3, 3x2 e 2x1 m, respectivamente (Quadro 3).

\section{Balanço de carbono por planta em cada área}

O balanço de carbono por planta foi superior no espaçamento de 3x3m e inferior no espaçamento $2 \times 1 \mathrm{~m}$ (Quadro 4)

\section{DISCUSSÃO}

A capacidade das florestas de acumular carbono na forma de biomassa (Xu et al., 2016) fazem com que tenham um papel importante na mitigação das mudanças climáticas (Matthies \& Valsta, 2016). Os plantios de eucalipto armazenam grande quantidade de carbono quando comparado com outras espécies florestais devido as altas taxas de crescimento (Maroni et al., 2017). O desenvolvimento da espécie nos primeiros anos é superior nos espaçamentos reduzidos (Lopes et al., 2017), porém com o passar dos anos, a maior competição por luz, nutrientes (Ghezehei et al., 2015) e menor taxa de mortalidade (Schumacher et al., 2011), faz com que os espaçamentos mais amplos consigam obter produtividades (Silva et al., 2016) e estocagem de carbono similares. O crescimento e maior volume de madeira das plantas tem relação direta com a estocagem de carbono, e são afetadas por fatores como qualidade do solo (Ueda et al., 2017), competição por luz (Coonen \& Sillett, 2015), resistência a seca (Hänke et al., 2016), temperatura do local (Chen et al., 2017) e a susceptibilidade a pragas e doenças (Bett et al., 2016).

O estoque de carbono nas áreas 1, 2 e 3 foi inferior ao observado em plantio de Eucalipto no espaçamento $3 \times 2$ m, aos 72 meses de idade, em Viçosa, MG (175,06 $\mathrm{MgCO}_{2 \mathrm{eq}} \mathrm{ha}^{-1}$ ) (Paixão et al., 2006). Essa diferença é justificada pois no inventário florestal foram contabilizados somente aqueles indivíduos com dap acima de $5 \mathrm{~cm}$, e no presente estudo todas aquelas presentes nas parcelas foram mensuradas. $\mathrm{O}$ estoque de carbono na área 4 foi inferior ao observado em plantio de Eucalipto na região central de Minas Gerais, no espaçamento $3 \times 2 \mathrm{~m}$, onde os resultados variaram de 50,06 a 98,94 $\mathrm{MgCO}_{2 \mathrm{eq}} \mathrm{ha}^{-1} \mathrm{ano}^{-1}$, devido ao teor de carbono utilizado $(0,5)$, superior ao do presente estudo, e terem sido considerados o estoque de carbono nos galhos e folhas (Gatto et al., 2011).

As emissões de GEE foram maiores nos menores espaçamentos. Isto acontece devido ao maior número de plantas por área, que exigiu uma maior fertilização

Quadro 2 - Áreas de plantio, espaçamento (em m), volume de madeira (em $\mathrm{m}^{3}$ ), densidade da madeira (em g $\mathrm{cm}^{3}$ ), estoque de carbono aos 32 meses (em MgCO2eq ha-1) e IMAC do plantio avaliado (em $\mathrm{MgCO}_{2 \text { eq }}$ ha $^{-1}$ ano $^{-1}$ )

\begin{tabular}{lccccc}
\hline Área & $\begin{array}{c}\text { Espaçamento } \\
(\mathrm{m})\end{array}$ & $\begin{array}{c}\text { Volume } \\
\left(\mathrm{m}^{3}\right)\end{array}$ & $\begin{array}{c}\text { Densidade } \\
\left(\mathrm{g} \mathrm{cm}^{3}\right)\end{array}$ & $\begin{array}{c}\text { Carbono } \\
\left(\mathrm{MgCO}_{2 \mathrm{eq}} \mathrm{ha}^{-1}\right)\end{array}$ & $\begin{array}{c}\text { IMAC } \\
\left(\mathrm{MgCO}_{2 \mathrm{eq}} \mathrm{ha}^{-1} \mathrm{ano}^{-1}\right)\end{array}$ \\
\hline 1 & $2 \mathrm{x} 1$ & 139,55 & 0,3778 & 90,94 & 33,69 \\
2 & $2 \mathrm{x} 2$ & 142,64 & 0,3289 & 80,96 & 29,98 \\
3 & $3 \times 2$ & 97,33 & 0,3318 & 55,71 & 20,63 \\
4 & $3 \times 3$ & 81,95 & 0,3302 & 46,68 & 17,29 \\
Média & & $115,37 \pm 30,39$ & $0,3422 \pm 0,02$ & $68,57 \pm 18,00$ & $25,39 \pm 6,68$ \\
\hline
\end{tabular}


Quadro 3 - Espaçamento (em m), emissões de GEE da fertilização nitrogenada e calagem (em $\mathrm{MgCO}_{2 \mathrm{eq}}$ ha ${ }_{-1}$ ), estoque de carbono (em MgCO2eq ha-1), balanço de carbono (em $\mathrm{MgCO}_{2 \mathrm{eq}}$ ha $^{-1}$ ) e emissões por $\mathrm{m}^{3}$ de madeira em $\left(\mathrm{MgCO}_{2 \mathrm{eq}} \mathrm{ha}^{-1}\left(\mathrm{~m}^{3}\right)^{-1}\right)$

\begin{tabular}{|c|c|c|c|c|c|}
\hline \multirow{2}{*}{$\begin{array}{l}\text { Espaçamento } \\
\text { (m) }\end{array}$} & \multicolumn{2}{|c|}{ Emissões $\left(\mathrm{MgCO}_{2 \mathrm{eq}} \mathrm{ha}^{-1}\right)$} & \multirow{2}{*}{$\begin{array}{c}\text { Estoque } \\
\left(\mathrm{MgCO}_{2 \mathrm{eq}} \mathrm{ha}^{-1}\right)\end{array}$} & \multirow{2}{*}{$\begin{array}{c}\text { Balanço } \\
\left(\mathrm{MgCO}_{2 \mathrm{eq}} \mathrm{ha}^{-1}\right)\end{array}$} & \multirow{2}{*}{$\begin{array}{l}\text { Emissões/Produção } \\
\left(\mathrm{MgCO}_{\text {2eq }} \mathrm{ha}^{-1}\left(\mathrm{~m}^{3}\right)^{-1}\right)\end{array}$} \\
\hline & Fertilização nitrogenada & Calagem & & & \\
\hline $2 \times 1$ & 0,88 & 0,24 & $+90,94$ & $+89,82$ & 0,0082 \\
\hline $2 \times 2$ & 0,44 & 0,24 & $+80,96$ & $+80,28$ & 0,0048 \\
\hline $3 \times 2$ & 0,33 & 0,24 & $+55,71$ & $+55,14$ & 0,0058 \\
\hline $3 \times 3$ & 0,22 & 0,24 & $+46,68$ & $+46,22$ & 0,0056 \\
\hline Média & $0,47 \pm 0,25$ & $0,24 \pm 0,00$ & $68,57 \pm 18,00$ & $67,80 \pm 17,78$ & $0,0061 \pm 0,0014$ \\
\hline
\end{tabular}

Quadro 4- Espaçamento (em m), número de plantas (em árv ha-1), emissões de GEE (em MgCO $_{2 \mathrm{eq}}$ ha $^{-1}$ planta-1 $^{-1}$ ), incremento médio anual em carbono (em $\mathrm{MgCO}_{2 \mathrm{eq}} \mathrm{ha}^{-1} \mathrm{ano}^{-1}$ planta $^{-1}$ ) e balanço de carbono por planta em cada um dos espaçamentos de plantio (em $\mathrm{MgCO}_{\text {2еq }}$ ha $^{-1}$ ano $^{-1}$ planta $^{-1}$ )

\begin{tabular}{ccccc}
\hline $\begin{array}{c}\text { Espaçamento } \\
(\mathrm{m})\end{array}$ & $\begin{array}{c}\text { Número de plantas } \\
\left(\text { árv ha }{ }^{-1}\right)\end{array}$ & $\begin{array}{c}\text { Emissões } \\
\left(\mathrm{MgCO}_{2 \mathrm{eq}} \text { ha } \mathrm{planta}^{-1}\right)\end{array}$ & $\begin{array}{c}\text { IMAC } \\
\left(\mathrm{MgCO}_{2 \mathrm{eq}} \text { ha }^{-1} \mathrm{ano}^{-1} \mathrm{planta}^{-1}\right)\end{array}$ & $\begin{array}{c}\text { Balanço } \\
\left(\mathrm{MgCO}_{2 \mathrm{eq}} \mathrm{ha}^{-1} \mathrm{ano}^{-1} \mathrm{planta}^{-1}\right)\end{array}$ \\
\hline 2x1 & 5000 & 0,0002 & 0,0067 & 0,0065 \\
2x2 & 2500 & 0,0002 & 0,0119 & 0,0117 \\
3x2 & 1667 & 0,0003 & 0,0124 & 0,0121 \\
3x3 & 1112 & 0,0004 & 0,0155 & 0,0151 \\
Média & & $0,0002 \pm 0,00009$ & $0,0116 \pm 0,0036$ & $0,0113 \pm 0,0035$ \\
\hline
\end{tabular}

e calagem, em relação aos outros espaçamentos. Quanto maior a quantidade de fertilizantes nitrogenados aplicados em plantios, maiores serão as emissões de óxido nitroso (Liu et al., 2017). Apesar disso, o maior número de plantas nos menores espaçamentos fez com que o balanço de carbono fosse mais positivo. O plantio de eucalipto continuará a aumentar seu balanço positivo de carbono ao longo dos anos até a sua estagnação de crescimento, pois não será realizada mais adubação. No longo prazo, é natural que as plantas compensem a libertação de carbono em estágios iniciais com o aumento da produtividade e sequestro de carbono.

O melhor balanço positivo por plantas no espaçamento 3x3 m é decorrente da menor competição por luz e nutrientes entre plantas, o que permite o melhor desenvolvimento das árvores. O espaçamento 2 x2 m foi o que obteve a menor emissão de GEE por $\mathrm{m}^{3}$ de madeira produzido. O maior número de mudas no espaçamento 2x1 m requer uma maior fertilização nitrogenada, e fez com que ele, apesar do maior estoque de carbono, obtivesse a maior emissão por $\mathrm{m}^{3}$ produzido. Esses plantios no espaçamento reduzido, são denominadas florestas energéticas (Couto et al., 2004), e são caracterizadas por árvores de pequeno diâmetro e com ciclo de corte inferior ao de espaçamentos mais amplos.

O balanço positivo em todos os sistemas enfatiza a importâncias das árvores como sumidouros de carbono e mitigadores das mudanças climáticas. Ainda que numa perspectiva, a abordagem de sistemas produtivos de baixo carbono contribui para a sustentabilidade local e, portanto, devem ser incentivados.

\section{CONCLUSÕES}

O estoque de carbono é superior no espaçamento de 2 x $1 \mathrm{~m}$. O balanço de carbono é positivo em todos os espaçamentos de plantio, sendo superior no espaçamento de $2 \times 1 \mathrm{~m}$, aos 32 meses. As florestas de eucalipto são capazes de neutralizar as emissões de gases de efeito estufa oriundas da fertilização nitrogenada e calagem, em plantios pouco tecnificados. A menor emissão por $\mathrm{m}^{3}$ de madeira produzido é no espaçamento de 2 × 2 m. É necessário acompanhar o estoque de carbono em idades avançadas do plantio, para avaliar a dinâmica de carbono nos diferentes espaçamentos ao longo dos anos. 


\section{LITERATURACITADA}

ALVARES, C.A.; STAPE, J.L.; SENTELHAS, P.C. et al. Köppen's climate classification map for Brazil. Meteorologische Zeitschrift, v.22, n.6, p.711-728, 2013.

Associação Brasileira de Normas Técnicas. (2003) - NBR 11941: determinação da densidade básica da madeira. Rio de Janeiro.

BETT, P.K.; DENG, A.L.; OGENDO, J.O. et al. Chemical composition of Cupressus lusitanica and Eucalyptus saligna leaf essential oils and bioactivity against major insect pests of stored food grains. Industrial Crops and Products, v.82, p.51-62, 2016.

BOUILLET, J.P.; LACLAU, J.P.; DE MORAES GONÇALVES, J.L. et al. Eucalyptus and Acacia tree growth over entire rotation in single-and mixed-species plantations across five sites in Brazil and Congo. Forest Ecology and Management, v.301, p.89-101, 2013.

CHEN, Y.; WANG, X.; JIANG, B. Tree survival and growth are impacted by increased surface temperature on paved land. Landscape and Urban Planning, v.162, p.68-79, 2017.

COONEN, E.J.; SILLETT, S.C. Separating effects of crown structure and competition for light on trunk growth of Sequoia sempervirens. Forest Ecology and Management, v.358, p.26-40, 2015.

COELHO, D.J.S.; SILVA, A.L.; SOARES, C.P.B. et al. Análise documental e de consistência técnica de planos de manejo florestal em áreas de formações florestais, no estado de Minas Gerais. Revista Árvore, v.32, n.1, p.69-80, 2008.

COUTO, L.C.; COUTO, L.; WATZLAWICK, L.F. et al. Vias de valorização energética da biomassa. Biomassa \& Energia, v.1, n.1, p.71-92, 2004.

GATTO, A.; FÉLIX DE BARROS, N.; NOVAIS, R.F. et al. Estoque de carbono na biomassa de plantações de eucalipto na região centro-leste do estado de Minas Gerais. Revista Árvore, v.35, n.4, p.895-905, 2011.
GHEZEHEI, S.B.; EVERSON, C.S.; ANNANDALE, J.G. Can productivity and post-pruning growth of Jatropha curcas in silvopastoral systems be regulated by manipulating tree spacing/ arrangement without changing tree density? Biomass and Bioenergy, v.74, p.233-243, 2015.

GHEZLOUN, A.; SAIDANE, A.; MERABET, H. The COP 22 New commitments in support of the Paris Agreement. Energy Procedia, v.119, n.1, p.10-16, 2017.

HÄNKE, H.; BÖRJESON, L.; HYLANDER, K. et al. Drought tolerant species dominate as rainfall and tree cover returns in the West African

Sahel. Land Use Policy, v.59, p.111-120, 2016.

Instituto Brasileiro de Árvores - IBA. Relatório da indústria brasileira de árvores 2017. In: http://iba.org/images/shared/Biblioteca/ IBA_RelatorioAnual2017.pdF (acessado em 29 de julho de 2017).

IPCC - Intergovernmental Panel on Climate Change - Guidelines for National Greenhouse Gas Inventories, Prepared by the National Greenhouse Gas Inventories Programme, Eggleston H.S., Buendia L., Miwa K., Ngara T. and Tanabe K. (eds). Published: IGES, Japan, 2006.

KUNHIKRISHNAN, A.; THANGARAJAN, R.; BOLAN, N.S. et al. Chapter One-Functional Relationships of Soil Acidification, Liming, and Greenhouse Gas Flux. Advances in Agronomy, v.139, p.1-71, 2016.

LIU, S.; WANG, J. J.; TIAN, Z. et al. Ammonia and greenhouse gas emissions from a subtropical wheat field under different nitrogen fertilization strategies. Journal of Environmental Sciences, v.57, p.196-210, 2017.

LOPES, E.D.; LAIA, M.L.; SANTOS, A.S. et al. Influência do espaçamento de plantio na produção energética de clones de Corymbia e Eucalyptus. Floresta, v.47, n.1, p.95-104, 2017.

MATTHIES, B.D.; VALSTA, L.T. Optimal forest species mixture with carbon storage and albedo effect for climate change mitigation. Ecological Economics, v.123, p.95-105, 2016. 
MORONI, M.T.; MUSK, R.; WARDLAW, T.J. Forest succession where trees become smaller and wood carbon stocks reduce. Forest Ecology and Management, v.393, p.74-80, 2017.

NEEFF, T. How many will attend Paris? UNFCCC COP participation patterns 1995-2015.

Environmental Science \& Policy, v.31, n.1, p.157-159, 2013.

PAIXÃO, F.A.; SOARES, C.P.B.; JACOVINE, L.A.G. et al. Quantificação do estoque de carbono e avaliação econômica de diferentes alternativas de manejo em um plantio de eucalipto. Revista Árvore, v.30, n.3, p.411-420, 2006.

PROIETTI, P.; SDRINGOLA, P.; BRUNORI, A. Assessment of carbon balance in intensive and extensive tree cultivation systems for oak, olive, poplar and walnut plantation. Journal of Cleaner Production, v.112, p.2613-2624, 2016.

\section{REPÚBLICA FEDERATIVA DO BRASIL.}

Pretendida contribuição nacionalmente determinada para consecução do objetivo da convenção-quadro das nações unidas sobre mudança do clima. In: http://www.itamaraty.gov.br/ images/ed_desenvsust/BRASIL-iNDCportugues.pdf (acessado em 29 de julho de 2017).

RIAHI, K.; KRIEGLER, E.; JOHNSON, N. et al. Locked into Copenhagen pledges - Implications of short-term emission targets for the cost and feasibility of long-term climate goals.

Technological Forescasting and Social Change, v.90, part A, n.1, p.8-23, 2015.
SCHUMACHER, M.V.; WITSCHORECK, R.; NEVES CALIL, F. Biomassa em povoamentos de Eucalyptus spp. de pequenas propriedades rurais em Vera Cruz, RS. Ciência Florestal, v.21, n.1, p.17-22, 2011.

SILVA, R.S.; VENDRUSCOLO, D.G.S.; ROCHA, J.R.M. et al. Desempenho silvicultural de Tectona grandis L. f. em diferentes espaçamentos em Cáceres,

MT. Floresta e Ambiente, v.23, n.3, p.397-405, 2016.

SUSAETA, A.; ADAMS, D.C.; GONZALES-

BENECKE, C. Economic vulnerability of southern US slash pine forests to climate change. Journal of Forest Economics, v.28, n.1, p.18-32, 2017.

TROTSIUK, V.; SVOBODA, M.; WEBER, P. et al. The legacy of disturbance on individual tree and standlevel aboveground biomass accumulation and stocks in primary mountain Picea abies forests. Forest Ecology and Management, v.373, n.1, p.108-115, 2016.

UEDA, M.U.; KACHINA, P.; MAROD, D. et al. Soil properties and gross nitrogen dynamics in old growth and secondary forest in four types of tropical forest in Thailand. Forest Ecology and Management, v.398, n.1, p.130-139, 2017.

VIERA, M.; SCHUMACHER, M.V.; TRÜBY, P. et al. Nutritional implications based in different intensities of Eucalyptus urophylla x Eucalyptus globulus biomass harvesting. Ciência Rural, v.45, n.3, p.432439, 2015.

XU, B.; PAN, Y.; PLANTE, A. F et al. Decadal change of forest biomass carbon stocks and tree demography in the Delaware River Basin. Forest Ecology and Management, v.374, n.1, p.1-10, 2016. .

Recebido para publicação em 13/07/2018 e aprovado em 15/03/2019 